developed a diagnostic strip and magnetic detector that, together with a smartphone, can diagnose hepatitis B in minutes using magnetically tagged biomarkers.

"It was an exhilarating journey to participate in the XCHALLENGE," Wang says. "The very challenging requirements posed by the XPRIZE Foundation forced us to design our assay and platform for the real world rather than the ivory tower. Being able to highlight novel nanomaterials such as magnetic nanoparticles and giant magnetoresistive (GMR) sensors in this international competition was also very rewarding."

Participants in such challenge prize competitions fund their entries through investors, crowdsourcing, institutional support, corporate sponsorship, philanthropic organizations, or their own wallets. In some cases, finalists receive seed money or a portion of the prize money. Organizers say this funding model provides better returns on their investment since the development costs are widely shared. Competitors in challenge prizes say this model involves more risk, but can lead to deep levels of commitment and productive new partnerships.

Participating in the USD\$1 million " $\mathrm{H} 2$ Refuel" H-Prize competition has led to new business ventures for SimpleFuel, the only finalist group in an ongoing DOE competition to develop home or community hydrogen refueling systems for fuel-cell electric vehicles. SimpleFuel is a consortium of companies with unique contributions, according to Darryl Pollica, team spokesperson and President and CEO of Ivys Inc., one of the member companies. "Our business arrangement will undoubtedly live beyond the competition as we already have aggressive plans to commercialize the hydrogen refueling appliance after the initial competition demonstration."

Credit for the earliest successful challenge competition usually goes to Britain's 1714 Longitude rewards for methods to determine a ship's longitude while at sea (see MRS Bulletin 25 [4], [2000], doi:10.1557/ mrs2000.51). The Ansari XPRIZE propelled challenge prizes into the modern spotlight in 2004, when Mojave Aerospace Ventures received a USD\$10 million prize for launching a reusable, manned spacecraft into space twice within a span of two weeks. Since then the idea has regained popularity primarily in the United States and Europe.

Challenge prizes based outside of the United States and Europe are difficult to find, but corporations, governments, and
Connect with open challenges

- ec.europa.eu/research/horizonprize

- www.Challenge.gov

- www.InnoCentive.com

- www.Kaggle.com

- www.NineSigma.com

- www.openIDEO.com

- www.xprize.org

foundations across the world are taking notice of their results and showing interest, according to Zenia Tata, executive director of international expansion and global development for XPRIZE. Digital platforms such as NineSigma.com and InnoCentive.com are becoming popular ways to connect visitors worldwide with open challenges from a range of hosts for varying prize amounts.

The rise in challenge prizes has caused a growing interest in studying their effectiveness. Initial reviews by McKinsey \& Company and others show that welldesigned contests can spur innovation in areas that are beyond the scope of traditional funding mechanisms, but exactly how they will affect the landscape of science research and advancement in the long term remains to be seen.

\section{XPRIZE provides incentive for radical breakthroughs in innovation}

\section{www.xprize.org By Paul Bunje, Jyotika Virmani, and Marcius Extavour}

$\mathrm{W}$ e are living in an era of extraordinary disruption. The technological, sociopolitical, and economic changes taking place around the world present innovators with an opportunity to apply groundbreaking research to challenges of worldwide importance.

Technologies on an exponential growth path (such as advanced robotics, ubiquitous sensors, synthetic biology) are rapidly becoming a part of our daily lives. These exponential technologies have the potential to lead to innovative solutions to some of the world's grand challenges. Importantly, many of these exponential technologies critically depend upon advances in materials science. Advanced materials are at the heart of innovative solutions to many of the world's biggest problems and opportunities.

The XPRIZE Foundation relies on the growing power of exponential technologies and revolutionary science to catalyze radical breakthroughs. By offering

Paul Bunje is principal scientist and senior director of Energy \& Environment at XPRIZE Jyotika Virmani is senior director of Energy \& Environment at XPRIZE.

Marcius Extavour is director of technical operations of the NRG COSIA Carbon XPRIZE.

a suite of incentives, XPRIZE seeks to inspire the world's scientists, technologists, and innovators to tackle seemingly intractable challenges.

"Grand Challenges" are a part of today's dynamic period of disruption. It is now possible for us to not only characterize massive, global threats and opportunities that might affect billions of people, but also to conceive of possible solutions. Listing grand challenges - poverty, climate change, a cure for cancer, total planetary exploration - may sound trivial, but in reality, framing a grand challenge requires understanding the complexity and nuances involved in both defining a 